\title{
Necklace-Ring Vector Solitons
}

\author{
Anton S. Desyatnikov and Yuri S. Kivshar \\ Nonlinear Physics Group, Research School of Physical Sciences and Engineering, \\ The Australian National University, Canberra ACT 0200, Australia \\ (Received 27 December 2000; published 27 June 2001)
}

\begin{abstract}
We introduce novel classes of optical vector solitons that consist of incoherently coupled self-trapped "necklace" beams carrying zero, integer, and even fractional angular momentum. Because of the stabilizing mutual attraction between the components, such stationary localized structures exhibit quasistable propagation for much larger distances than the corresponding scalar vortex solitons and expanding scalar necklace beams.
\end{abstract}

DOI: $10.1103 /$ PhysRevLett.87.033901

PACS numbers: 42.65.Tg, 41.20.Jb

Spatial optical solitons (or self-trapped optical beams) have attracted significant attention during the last few years due to an impressive experimental progress in generating such beams in different types of nonlinear bulk media [1]. Since the pioneering paper by Chiao et al. [2], the self-trapped optical beam is viewed as a guided mode of the effective waveguide it induces in a nonlinear medium, and such beams are usually associated with the fundamental guided modes (i.e., the modes without nodes). Nevertheless, in the earlier days of nonlinear optics, it was noticed that the light self-trapping may also occur for higher-order beams of a radial symmetry [3]. More recently, the self-trapped azimuthally periodically modulated beams, "necklace-ring" beams, were shown to exhibit quasistable propagation in a self-focusing Kerr medium $[4,5]$. One of the main properties of the necklace-ring beams is their ability to transport several times the critical power of the fundamental beam in a nonlinear Kerr medium, known itself to exhibit catastrophic collapse (or blowup) phenomenon. However, the necklace-ring beams do not exist as stationary self-trapped structures, and they expand with propagation because adjacent bright "petals" on the ring structure differ in phase by $\pi$ and, therefore, the neighboring beamlets repel each other [4].

The repulsion and subsequent diffraction of the neighboring petals of the self-trapped necklace beams are the main physical mechanisms of their disintegration. In this Letter, we show that the incoherent interaction between the components of a composite (or vector) ringlike beam allows one to compensate for repulsion of beamlets, thus creating a new type of stationary self-trapped structures exhibiting the properties of the necklace-ring beams. The physical mechanism for creating such vector ring solitons is somewhat similar to the mechanism responsible for the formation of the solitonic gluons [6] and multihump vector solitary waves [7], and it is explained by a balance of the interaction forces acting between the coherent and incoherent components of the composite solitons.

To introduce the concept of vector ring solitons, we consider the interaction of $N$ mutually incoherent optical beams propagating in a self-focusing bulk nonlinear medium. Equations for the slowly varying beam en- velopes $E_{n}(x, y, z)$ can be written in the form of the normalized coupled nonlinear Schrödinger (NLS) equations $(n=1,2, \ldots, N)$,

$$
i \frac{\partial E_{n}}{\partial z}+\frac{\partial^{2} E_{n}}{\partial x^{2}}+\frac{\partial^{2} E_{n}}{\partial y^{2}}+f(I) E_{n}=0,
$$

where the transverse, $(x, y)$, and propagation, $z$, coordinates are measured in the units of $\left(L_{D} / k\right)^{1 / 2}$ and $L_{D}$, respectively, $L_{D}$ being the diffraction length. Function $f(I)$ describes the nonlinear properties of an optical medium, and it is assumed to depend on the total beam intensity, $I=\sum\left|E_{n}\right|^{2}$.

For $N=1$ (a scalar soliton), the well-known localized solutions carrying an angular momentum are vortices with the following structure: $E=u(r) \exp (i m \varphi+i k z)$, where $r=\sqrt{x^{2}+y^{2}}$ and $\varphi=\tan ^{-1}(y / x)$. In order to establish a link between the vortices and necklace beams, we note that the vortex can be presented as a coherent superposition of two necklacelike beams, $E=\mathcal{E}_{1}+\mathcal{E}_{2}$, where

$$
\mathcal{E}_{1,2}=u(r)\left\{\begin{array}{c}
\cos (m \varphi) \\
i \sin (m \varphi)
\end{array}\right\} e^{i k z} .
$$

The purpose of this Letter is to construct novel types of localized solutions of the NLS equations (1) using an incoherent superposition of the necklace-type components. We look for the solutions in the form

$$
E_{n}=u(r) \Phi_{n}(\varphi) e^{i k z}
$$

with the self-consistency condition $\sum\left|\Phi_{n}(\varphi)\right|^{2}=1$. The total intensity of such a localized mode is a function of the radial coordinate only, $I=u^{2}(r)$, and the components of the ring vector soliton $E_{n}$ complement each other creating a perfect ring structure, however, possessing no radial symmetry by themselves. As we demonstrate below, such composite solitary waves display the properties of both vortices and necklace-ring beams being, however, much more robust in the propagation.

To find the structure of the vector ring solitons, we substitute Eq. (2) into Eq. (1) and obtain 


$$
\begin{aligned}
\frac{d^{2} u}{d r^{2}}+\frac{1}{r} \frac{d u}{d r}-\frac{m^{2}}{r^{2}} u-k u+f\left(u^{2}\right) u & =0 \\
\frac{d^{2} \Phi_{n}}{d \varphi^{2}}+m^{2} \Phi_{n} & =0
\end{aligned}
$$

Solutions of the first equation of the system (3) can be found numerically as a discrete set of localized modes with nodes, whereas the latter equation has an analytical solution $\Phi_{n}(\varphi)=a_{n} \cos (m \varphi)+b_{n} \sin (m \varphi)$, with integer $m$ and the complex coefficients $a_{n}$ and $b_{n}$ satisfying the conditions

$$
\sum \operatorname{Re}\left(a_{n} b_{n}^{*}\right)=0, \quad \sum\left|a_{n}\right|^{2}=\sum\left|b_{n}\right|^{2}=1 .
$$

Equations (4) define exact solutions of the system (1) for any $N$ and, in the particular case $N=1$, they describe a scalar vortex with $a=1$ and $b=i$.

Localized solutions of this kind are characterized by the partial powers of the soliton components,

$$
P_{n}=\int\left|E_{n}\right|^{2} d \vec{r}=\frac{1}{2}\left(\left|a_{n}\right|^{2}+\left|b_{n}\right|^{2}\right) P^{(m)},
$$

where $d \vec{r}=r d r d \varphi, \quad P^{(m)}=2 \pi \int_{0}^{\infty} u^{2}(r) r d r$ is the power of a scalar vortex with the topological charge $m$, and the total power is $P_{\text {tot }} \equiv \sum P_{n}=P^{(m)}$.

The most important characteristic of the vector ring soliton is its angular momentum $M=\sum M_{n}$, where

$$
M_{n}=\int \operatorname{Im}\left(E_{n}^{*} \frac{\partial E_{n}}{\partial \varphi}\right) d \vec{r}=m \operatorname{Im}\left(a_{n}^{*} b_{n}\right) P^{(m)}
$$

A ratio of the total angular momentum of the vector ring soliton to its total power, $M / P^{(m)}$, can be regarded as an analog of "spin" of an optical beam. In particular, for the vector vortices (when $b_{n}= \pm i a_{n}$ ) this ratio is zero or integer, $M / P^{(m)}=m$. However, in a general case described by Eq. (5), this value is not necessarily integer and, therefore, the vector ring solitons and its components can possess a fractional value of the spin. Such an unusual result is a direct manifestation of the wave phase which is a nonlinear function of the polar angle $\varphi$, and it describes a screw dislocation of the soliton wave front. Such a case resembles the recently studied modulated scalar necklace beams [5]; however, in our case we suggest, for the first time to our knowledge, stationary localized structures carrying a fractional angular momentum (see discussions below).

We have identified several different classes of exact localized solutions described by Eqs. (1). In order to test their robustness to the propagation, we have performed a series of numerical simulations for a two-component model $(N=2)$, also selecting a saturable nonlinearity $f(I)=I /(1+s I)$ in order to eliminate collapse. Such a saturable nonlinearity describes, in a greatly simplified isotropic approximation, the screening spatial solitons in photorefractive optical materials. In all simulations discussed below we select the saturation parameter $s=0.5$, since this value is closer to the experimental situation.
First, we analyze the simplest solution of this type that has equal powers in each component, $P_{1,2}=\frac{1}{2} P^{(m)}$, and is characterized by the amplitudes

$$
\begin{aligned}
& a_{1}=\left(1+p^{2}\right)^{-1 / 2}, \quad b_{1}=i p a_{1}, \\
& a_{2}=p a_{1}, \quad b_{2}= \pm i a_{1},
\end{aligned}
$$

with real $p \in[0 ; 1]$. We start from the solution (6) with $m=1$ which, for $p=0$, represents an incoherent superposition of two dipole modes. Such a localized solution displays a long-term stable dynamics up to the propagation distances of almost 55 diffraction lengths, then it exhibits a symmetry-breaking instability and decays into three isolated vector solitons that fly away along the radial directions [see Fig. 1(b)].

For comparison, in Fig. 1(a) we present the corresponding breakup of a scalar vortex with $m=1$. In spite of the fact that the total beam intensities in both cases are absolutely identical, the splitting dynamics presented in Figs. 1(a) and 1(b) are remarkably different. In the former case, the vortex decays into a pair of scalar solitons with transformation of the vortex angular momentum into the linear momenta of the partial solitons flying away along the tangential directions [8]. In contrast, the vector ring soliton in Fig. 1(b) has a planar wave front and a zero spin, and the soliton trajectories have only normal components.

A common feature observed for all vector ring solitons with zero spin is that their breakup occurs with a splitting into an odd number of filament solitons, unlike an even number of solitons produced by the symmetry-breaking instability of scalar vortex solitons. Thus, the symmetrybreaking instability of the vortex ring solitons with zero total spin is accompanied by the birth of additional solitons. We note also that the intensities of each of the components
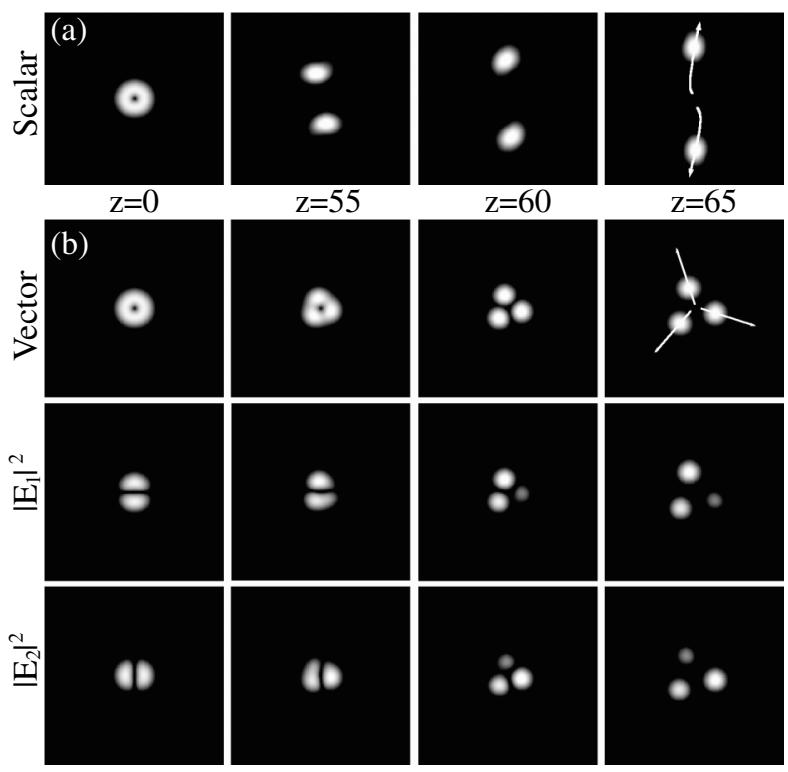

FIG. 1. Dynamics and symmetry-breaking instability of the ring solitons with $m=1$ : (a) scalar vortex and (b) vector ring soliton (6) for $p=0$. Arrows show the trajectories of the flying solitons after the instability-induced splitting. 
are symmetrically modulated, as clearly seen in Fig. 1(b), but the total beam intensity is uniform.

Self-trapped localized structures consisting of a large number of petals, or necklace-ring beams, exhibit a strong effective stabilization even for the Kerr medium [4]. We observe similar features for the vector necklace-ring solitary waves, and in Figs. 2 and 3 we present the dynamics of the vector solitons for $m=6$. For comparison, we show as well the expansion of a scalar necklace beam with 12 lobes and the breakup of a scalar vortex into eight filament beams [see Figs. 2(a) and 2(b)].

To compare the beam dynamics and instability-induced splitting in all those cases, we introduce, for the necklace beams, the propagation distance when the necklace increases its radius by the factor of 2 , the so-called expansion length $L_{e}$. The vortex dynamics is characterized by the propagation distance on which its breakup occurs, the splitting length $L_{s}$. For the dynamics of the necklace beam created by the input $E(z=0)=u(r) \cos (6 \varphi)$, we find $L_{e} \simeq 50$, whereas for a scalar vortex, $L_{s} \simeq 35$ (see Fig. 2).

As is shown in Fig. 2(c), the vector necklace-ring soliton breaks up into 15 filaments with a ratio of the component powers modulated along the ring. Corresponding physical parameters are the splitting distance of the ring, $L_{s} \simeq 55$, and the distance at which the ring structure becomes twice as big, $L_{e} \simeq 110$. A comparison shows that an effective

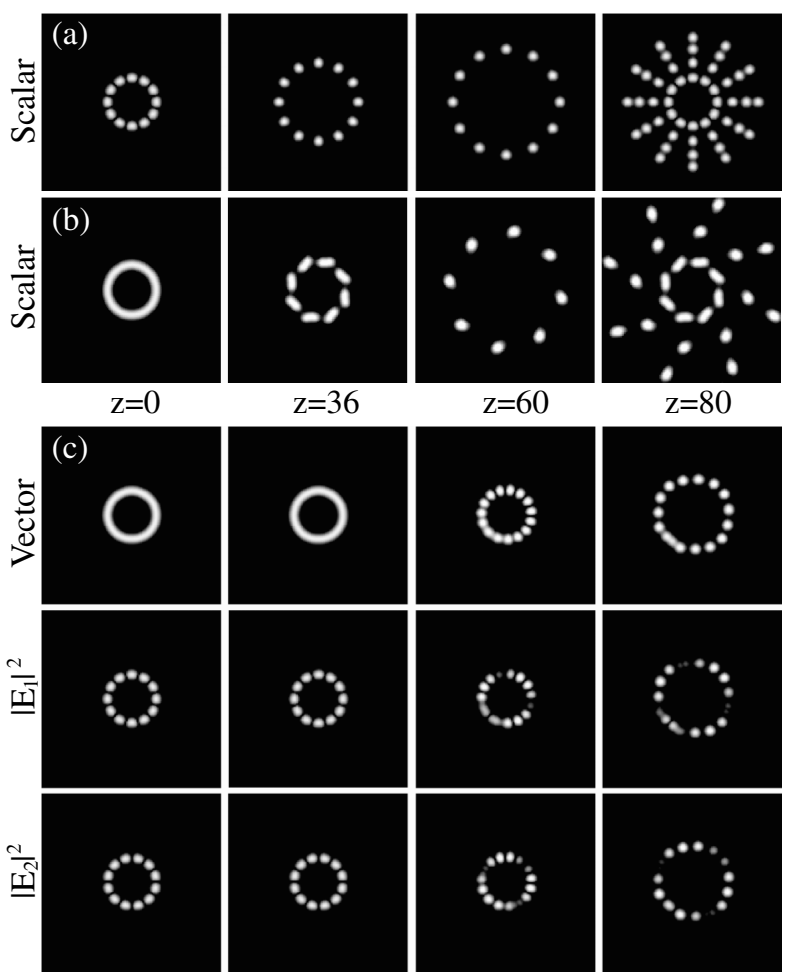

FIG. 2. Evolution of three types of ring beams at $m=6$ : (a) scalar necklace beam, $E(z=0)=u(r) \cos (6 \varphi)$; (b) scalar vortex, $E(z=0)=u(r) \exp (i 6 \varphi)$; and (c) vector ring soliton (6) for $p=0$. The frames in (a),(b) at $z=80$ show a superposition of the structures at different propagation distances. "lifetime" $L_{s}$ of the vector ring soliton with $m=6$ is up to $60 \%$ larger than that of the corresponding scalar vortex, and the expansion of the vector ring soliton is 2 times slower than that of the scalar necklace beam. Although expansion of scalar necklace beams decreases significantly with an increasing of the number of petals [4], we use this value for comparison, as we observe the similar behavior for vector necklaces.

For $p \neq 0$, we have two types of localized solutions corresponding to two different signs of $b_{2}$ in Eqs. (6). Both of them have equivalent component envelopes and powers but different values of the total momentum $M=M_{1}+M_{2}$,

$$
M_{1}= \pm M_{2}=\frac{m p}{1+p^{2}} P^{(m)} .
$$

While one of these solutions has a zero angular momentum, the total spin of the other solution is nonzero, $M=2 M_{1}$, and it can be fractional. The dynamics of two such solutions differ dramatically, as shown in Figs. 3(a) and $3(\mathrm{~b})$. For the vector ring soliton with fractional spin $M / P^{(6)}=5.76$ [see Fig. 3(a)], a centrifugal force dominates and the ring structure breaks similarly to the vortex breakup [cf. Fig. 2(b)]. Dynamics of the solution
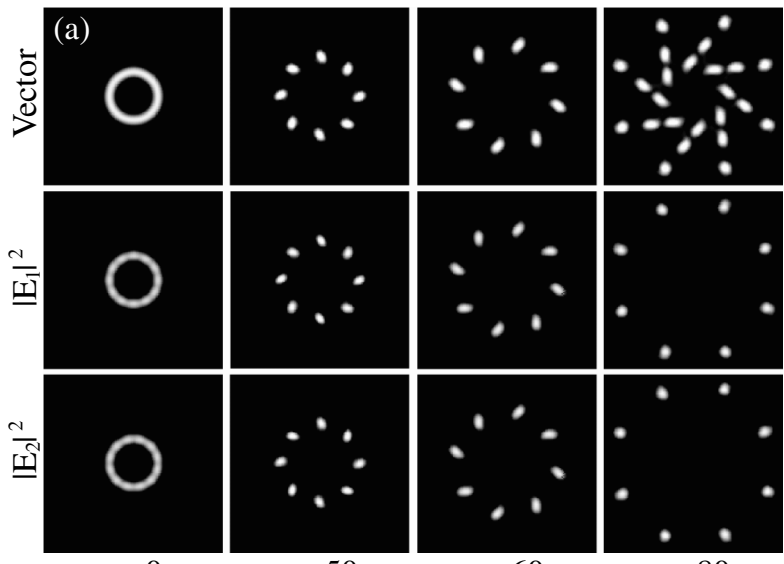

$\mathrm{Z}=50$

$\mathrm{z}=60$

$\mathrm{z}=80$
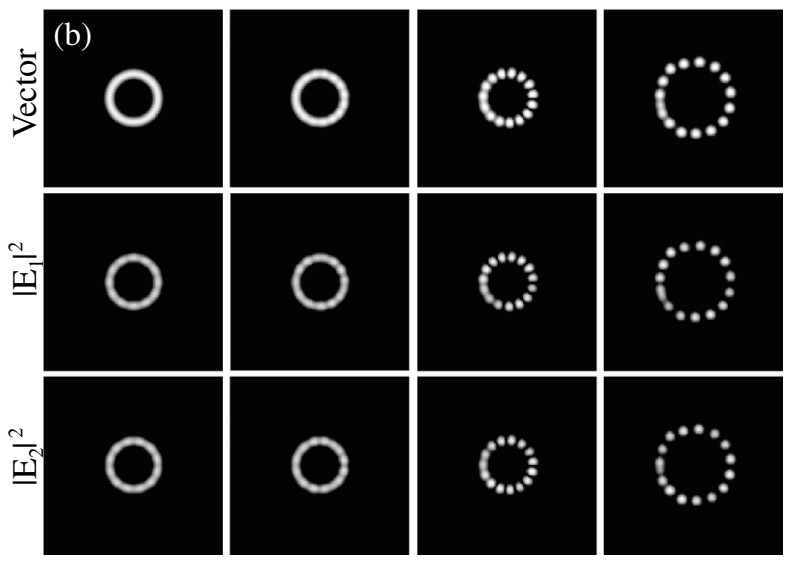

FIG. 3. Evolution of the vector ring solitons with a nonzero spin in each component, $m=6$ and $p=0.75$ [see Eqs. (6)]. (a),(b) Intensities of the vector rings with nonzero and zero total spin, respectively. 
with zero total spin follows a different scenario, as shown in Fig. 3(b) [cf. Fig. 2(c)], and it represents a type of the symmetry-breaking instability when the interaction and mutual guiding of filaments produce new solitons.

We emphasize that two different scenarios of the instability-induced dynamics presented in Figs. 3(a) and 3(b) are governed by the total (zero or nonzero) spin of the structure. For small values of $p$, the ring soliton with a fractional spin breaks up into vector solitons of different sizes. Since a vector soliton with a lower power has a larger radial velocity, the whole structure expands with rotation, but it does not preserve its radial symmetry.

For larger values of the parameter $p$, the components of the vector ring soliton change their structure from the necklace type to the full ring, and for $p=1$ such a solution reduces to a two-component vortex ring with, respectively, zero or integer spin $m$. Such a transformation is seen as a change of the modulation depth of the ringlike components of the vector solitons [cf. Figs. 2(c) and 3(b)]. As a result, the decay scenario changes as well and at $p=1$, the vector vortex with zero total spin breaks up into 15 filaments with equal powers in the components.

Another interesting structure described by Eqs. (3) carries different powers and spins in each of the components, and its parameters are

$$
\begin{gathered}
a_{1}=1, \quad b_{1}=i q, \quad a_{2}=0, \quad b_{2}=\sqrt{1-q^{2}}, \\
P_{1,2}=\frac{1}{2}\left(1 \pm q^{2}\right) P^{(m)}, \quad M_{1}=m q P^{(m)}, \quad M_{2}=0 .
\end{gathered}
$$

Here the parameter $q \in[0 ; 1]$, and in the limit $q=0$ this solution coincides with Eqs. (6) for $p=0$. For $q$ close to 1 , the main features of the symmetry-breaking instability discussed above remain valid, but the dynamics becomes more complicated, due to a strong guiding effect in the regime where $P_{1} \gg P_{2}$. In the limit $q=1$, the localized solution (7) describes a scalar vortex in the first mode, similar to that shown in Figs. 1(a) and 2(b).

A strong stabilizing effect of the incoherent interaction of the azimuthally modulated beams can also be observed for higher-order localized modes. In particular, we show one of the examples in Fig. 4(a), where a scalar doublering vortex is completely destroyed at the distance $z=50$ while the similar vector structure, but with zero spin, still presents its ringlike shape [see Fig. 4(b)].

The scalar "necklace" beams have been observed experimentally as expanding structures with a large number of petals [9]. Vectorial beam stabilization has been recently observed in photorefractive crystals at $\mu \mathrm{W}$ powers for the double-dipole beams shown in Fig. 1(b) [10] and also for the beams consisting of four and six beamlets [11]. Composite beams were created in the way similar to the multipole spatial solitons [12], by employing the phase-imprinting technique. Although the photorefractive nonlinearity is anisotropic and nonlocal, an effective vectorial stabilization has been proved to be the key
Scalar

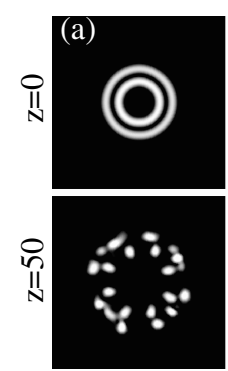

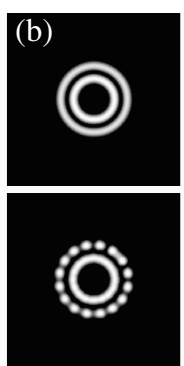

Vector

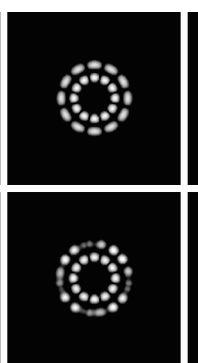

$\left|\mathrm{E}_{1}\right|^{2}$

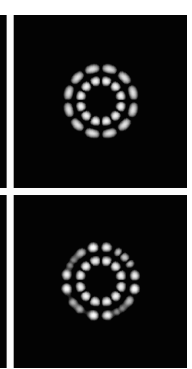

$\left|\mathrm{E}_{2}\right|^{2}$
FIG. 4. Two-ring solitons with $m=6$. (a) Scalar higher-order vortex and (b) vector double-ring soliton.

mechanism that allows one to generate quasistationary composite light beams with modulated components.

In conclusion, we have presented novel classes of stationary optical beams in the form of vector ring spatial solitons that consist of necklace-type components. Such vector solitons exhibit quasistable propagation for much larger distances than the corresponding vortex solitons and scalar necklace beams, demonstrating the features of both those types of self-trapped ringlike structures. Similar to the scalar vortex solitons, the vector ring solitons exhibit the symmetry-breaking instability that is accompanied by a transformation of the (integer or fractional) angular momentum into the linear momenta of the individual soliton beamlets flying away. Our approach may also be useful for other problems, e.g., for the study of a mixture of several Bose-Einstein condensates trapped by a parabolic potential, or for the dynamics of "braided light" in plasmas [13].

The authors are indebted to W. Krolikowski, D. Neshev, M. Segev, M. Soljačić, and G. I. Stegeman for useful discussions and E. Ostrovskaya for help with numerical simulations and a critical reading of this manuscript. The work was partially supported by the Performance and Planning Fund of the Australian National University.

[1] G. I. Stegeman and M. Segev, Science 286, 1518 (1999).

[2] R. Y. Chiao et al., Phys. Rev. Lett. 13, 479 (1964).

[3] H. A. Haus, Appl. Phys. Lett. 8, 128 (1966).

[4] M. Soljačić, S. Sears, and M. Segev, Phys. Rev. Lett. 81, 4851 (1998); see also M. Soljačić and M. Segev, Phys. Rev. E 62, 2810 (2000).

[5] M. Soljačić and M. Segev, Phys. Rev. Lett. 86, 420 (2001).

[6] E. A. Ostrovskaya et al., Opt. Lett. 24, 327 (1999).

[7] E. A. Ostrovskaya et al., Phys. Rev. Lett. 83, 296 (1999).

[8] W. J. Firth and D. V. Skryabin, Phys. Rev. Lett. 79, 2450 (1997); D. V. Skryabin and W. J. Firth, Phys. Rev. E 58, 3916 (1998).

[9] A. Barthelemy et al., Proc. SPIE Int. Soc. Opt. Eng. 2041, 104 (1994).

[10] M. Ahles, K. Motzek, A. Stepken, F. Kaizer, C. Weilnau, and C. Denz (unpublished).

[11] D. Neshev (private communication).

[12] A. Desyatnikov et al., Opt. Lett. 26, 435 (2001).

[13] C. Ren et al., Phys. Rev. Lett. 85, 2124 (2000). 